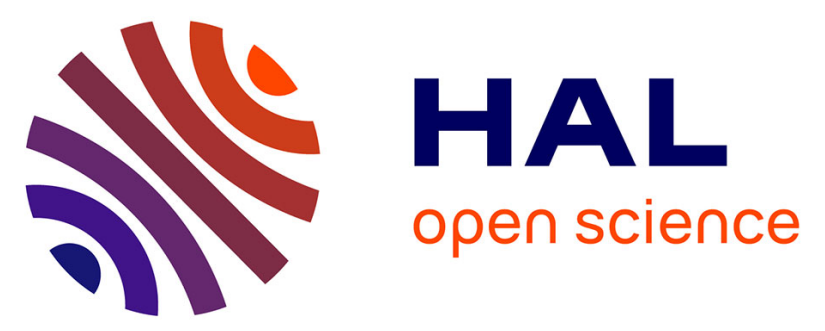

\title{
Tight-binding modeling of interstitial ordering processes in metals: Application to zirconium hydrides
}

Paul Eyméoud, Fabienne Ribeiro, Azzam Charaf-Eddin, Rémy Besson, Guy Treglia

\section{- To cite this version:}

Paul Eyméoud, Fabienne Ribeiro, Azzam Charaf-Eddin, Rémy Besson, Guy Treglia. Tight-binding modeling of interstitial ordering processes in metals: Application to zirconium hydrides. Physical Review B, 2020, 101 (22), 10.1103/PhysRevB.101.224106 . hal-03038040

\section{HAL Id: hal-03038040 https://hal.science/hal-03038040}

Submitted on 3 Dec 2020

HAL is a multi-disciplinary open access archive for the deposit and dissemination of scientific research documents, whether they are published or not. The documents may come from teaching and research institutions in France or abroad, or from public or private research centers.
L'archive ouverte pluridisciplinaire HAL, est destinée au dépôt et à la diffusion de documents scientifiques de niveau recherche, publiés ou non, émanant des établissements d'enseignement et de recherche français ou étrangers, des laboratoires publics ou privés. 


\title{
Tight-binding modeling of interstitial ordering processes in metals: Application to zirconium hydrides
}

\author{
Paul Eyméoud, ${ }^{1}$ Fabienne Ribeiro $\odot,{ }^{1}$ Azzam Charaf-Eddin, ${ }^{2}$ Rémy Besson, ${ }^{3}$ and Guy Tréglia ${ }^{2}$ \\ ${ }^{1}$ Institut de Radioprotection et de Sûreté Nucléaire, PSN-RES, SEMIA, LPTM, 13115 St Paul Lez Durance cedex, France \\ ${ }^{2}$ Groupe de Métallurgie Physique et Génie des Matériaux, Unité Matériaux et Transformations, CNRS UMR 8207, \\ Université de Lille 1, Bâtiment C6, 59655 Villeneuve d'Ascq cedex, France \\ ${ }^{3}$ Aix Marseille Université, CNRS, CINAM, Marseille, France
}

(Received 11 October 2018; revised manuscript received 11 February 2020; accepted 5 May 2020; published 5 June 2020)

\begin{abstract}
We present here a theoretical study of ordering processes in metal-hydrogen compounds based on a generalized perturbation method and on tight-binding coherent potential approximation. This approach is illustrated for zirconium hydrides, in which case we demonstrate that a cluster expansion of the ordering energy can be limited to effective pair interactions, the leading one being between hydrogen atoms in third-neighbor positions. These results are quantitatively confirmed by comparison to density functional theory calculations and qualitatively interpreted through orbital symmetry analysis. The method is then applied first to draw a preliminary $\mathrm{Zr}-\mathrm{H}$ phase diagram and then to characterize the effect of lattice deformation on the ordering processes in zirconium hydrides.
\end{abstract}

DOI: 10.1103/PhysRevB.101.224106

\section{INTRODUCTION}

The dream of modeling the behavior of simple materials (such as binary alloys) from their electronic structure took shape in the 1990s when ab initio calculations began to benefit from computer power. Since then, databases containing the energies of numerous ordered phases for specific binary alloys have been elaborated. One of their applications is to derive the equilibrium phase diagrams, not always experimentally known, via the coupling between $a b$ initio energy data and more or less refined statistical physics approaches. A wide domain of approaches, ranging from $a b$ initio to phase diagram calculations, was developed, aimed at improving the understanding and control of industrial and technological processes. This has, in particular, benefited from the development of the cluster expansion method (CEM) [1], which consists of expressing the energy of an ordered structure as a linear combination of many-body interactions (pairs, triplets, quadruplets, etc.). These effective cluster interactions are obtained from first-principles DFT calculations through the Connolly-Williams structure inverse method based on a mapping of total energies of a given set of predefined ordered structures onto a generalized Ising-type model [2]. Such a methodology opens up a very broad field in materials science, not only for metal alloys but also for mixed compounds such as hydrides and carbides.

Interstitial hydrides of $d$ and $f$ block metals constitute an important topic in material science, related to hydrogen storage in battery cells [3], and metal embrittlement [4]. To study these compounds at the atomistic scale, one needs to describe the numerous structures that can exist at fixed concentration and to take into account the effect of temperature $[5,6]$, which requires using thermostatistical approaches. The difficulty is then to get an atomistic energetic model that is accurate enough to treat ordering processes (hundredth of an $\mathrm{eV}$ accuracy) while remaining cheap in CPU time. For several transition-metal hydrides, the CEM has already furnished good results (Ti-H [6], Zr-H [7,8], La-H [9]). However, this approach remains questionable and limited. First, the manybody character of the energy should forbid using too many simple cluster expansions so that one needs to justify them from the electronic structure. Then, it requires postulating $a$ priori the order of preponderant interactions and the size of clusters to use. Finally, it implies performing a large number of density functional theory (DFT) calculations if one aims at fitting a given strained state.

The aim of the present paper is to calculate directly the cluster interactions involved in CEM in order to identify the relevant interactions to derive from DFT total energy calculations, what their range is, and what the leading terms are. An important point here is that these cluster interactions are per se both concentration and volume dependent. These interatomic interactions are obtained through a generalized perturbation method (GPM) which treats the ordering process as a perturbation of the disordered state. This GPM approach was first developed to study ordering processes in substitutional metallic alloys [10-14] by treating the electronic structure within the tight-binding (TB) formalism [15] and the disordered state with the self-consistent coherent potential approximation (CPA) approach [16,17]. The main result of these studies was that a truncation of the perturbative development to second order (i.e., to pairwise interactions) is sufficient to approximate ordering band energies and to characterize the relative stabilities of ordered structures at $0 \mathrm{~K}$.

This TB-GPM method was then extended to account for vacancy-carbon ordering in interstitial carbides of transition 
metals [18], showing that cluster expansion could still be truncated to pair interactions. The main difference between the substitutional and interstitial systems concerns the leading interactions, which are those between first neighbors in the former case and between second neighbors in the latter one.

The success of the original TB-GPM method then sparked a lot of studies in the alloy literature, among which are various attempts to put together a real-space way of solving the inhomogeneous CPA equations with recursion for both diagonal and off-diagonal disorder (Shiba approximation [19]) and extension to multicomponent alloys and transport (KuboGreenwood) [20-22]. Indeed, a main advantage of such a real-space approach is that it can be easily applied to finite systems (such as nanoalloys) or amorphous alloys such as NiZr [23].

Moreover, many authors aimed at implementing the TBGPM idea in an $a b$ initio context, first based on the Generalized Perturbation Method based on Korringa-Kohn-Rostoker Coherent Potential Approximation and later on Generalized Perturbation Method based on Tight-Binding Linear MuffinTin Orbitals Coherent Potential Approximation approaches [24-26]. It is worth noticing that one has to be careful when applying GPM to the possible charge effects. Indeed, these effects have often been neglected, leading to a less accurate description than structure inverse methods when they are important. To take them into account, Ruban et al. proposed to account for the screened Coulomb interaction that describes charge transfers within single-site approximation to DFT and the force theorem, showing that the resulting screened GPM interactions reproduce the configurational energy of a wide range of substitutional metallic alloys [27] and also interstitial carbides [28] and complex nitrides [29] and oxides [30]. In particular in the latter reference, the authors were able to determine the relevant interactions in CEM through a comparison of DFT screened Generalized Perturbation Method and Connolly-Williams interactions [30].

Despite this body of literature and advanced works, there is still a need to develop the original TB-CPA-GPM to address new systems and physical problems since the already quoted mixed alternative DFT-GPM approaches lead, in general, to the same results as TB-CPA-GPM without its flexibility. In this sense, the latter approach is far from being out of date and is still very efficient at giving access to driving forces and rationalizing modeling formalism choice. Thus, it remains useful to still develop and improve this kind of methodology in the aim to derive a general classification map of behavior based on systematic studies along lines and columns of the periodical table.

Here we illustrate this approach in the case of interstitial metallic hydrides, applied to the $\mathrm{Zr}-\mathrm{H}$ system, which constitutes a major topic in nuclear safety (zirconium-based nuclear fuel claddings can be embrittled by hydrides in a pressurized water reactor [31-33]). This approach can easily be extended to any nonmagnetic interstitial metallic hydride.

Since the majority of zirconium hydrides which appear in the binary $\mathrm{Zr}-\mathrm{H}$ diagram present a fcc or face-centeredtetragonal (fct) structure $[34,35]$, this system can be modeled as built on a $\mathrm{Zr}$ fcc lattice, with a tetrahedral interstitial sublattice occupied by hydrogen atoms $\mathrm{H}$ and vacancies $\square$ (stoichiometry $\mathrm{ZrH}_{c} \square_{2-c}$, where $c$ represents the hydrogen concentration). In this framework, the order and disorder phenomena exclusively concern this interstitial sublattice. Note that even though it has been established, both theoretically and experimentally, that the $\mathrm{H}$ atoms should be localized on the tetrahedral sites whatever the crystalline structure, hcp or fcc, the octahedral ones are found quite close in energy in the hcp structure. In this phase, these sites play an important role in hydrogen diffusion [36,37], hydride precipitation [5,38], and embrittlement $[39,40]$. Nevertheless, since we are concerned here only with equilibrium thermodynamics and not with kinetics, in the fcc structure, we can assume that all hydrogen atoms occupy only the tetrahedral sublattice.

This paper is organized as follows. First, a short description of the theoretical background of the TB-GPM is given, together with its numerical implementation for the $\mathrm{ZrH}_{c} \square_{2-c}$ system. Then the multiplet interactions are derived, showing that they are negligible beyond pair interactions. The latter, which are both concentration and volume dependent, are then validated quantitatively from the comparison with DFT calculations and qualitatively through orbital symmetry considerations. Finally, in order to illustrate the potentiality of this methodology, it is used, on the one hand, to draw a preliminary phase diagram of the tetrahedral interstitial sublattice of the fcc $\mathrm{ZrH}_{c} \square_{2-c}$ system as a function of the relevant interactions and, on the other hand, to characterize the effect of lattice deformation on the ordering processes.

\section{THEORETICAL BACKGROUNDS}

The electronic structure of the $\mathrm{ZrH}_{c} \square_{2-c}$ system is described within the tight-binding approximation [15], which writes the Hamiltonian (1) in the atomic orbital basis $\{i, \lambda\}$ (with $i$ being the site index and $\lambda$ being the orbital one), where the diagonal term $\epsilon_{i}^{\lambda_{u}}$ represents the energy of orbital $\lambda_{u}$ at atomic site $i$ and the nondiagonal term $\beta_{i j}^{\lambda_{u} \mu_{v}}$ is the hopping integral between the $\lambda_{u}$ orbital at atomic site $i$ and $\mu_{v}$ orbitals at atomic site $j$ :

$$
\begin{aligned}
H= & \sum_{i} \sum_{u} \sum_{\lambda_{u}} p_{i}^{u}\left|i, \lambda_{u}\right\rangle \epsilon_{i}^{\lambda_{u}}\left\langle i, \lambda_{u}\right| \\
& +\sum_{\substack{u, v \\
\sum_{i, j}}} \sum_{\lambda_{u}, \mu_{v}} p_{i}^{u} p_{j}^{v}\left|i, \lambda_{u}\right\rangle \beta_{i j}^{\lambda_{u} \mu_{v}}\left\langle j, \mu_{v}\right| .
\end{aligned}
$$

In this formulation, the $u$ and $v$ indexes label the chemical nature of the atom, which can take three values: $\mathrm{Zr}$ for a zirconium atom, $\mathrm{H}$ for a hydrogen atom, and $\square$ for an interstitial tetrahedral atomic vacancy. The Hamiltonian depends on the configuration through the $p_{i}^{u}$ factor, which corresponds to an atomic occupation number equal to 1 if site $i$ is occupied by an atom of type $u$ and 0 otherwise. The interstitial nature of the alloy $\mathrm{ZrH}_{c} \square_{2-c}$ is accounted for by preventing electrons from hopping on vacancy sites, as suggested by Faulkner to treat palladium hydrides [17]. This means that the energy levels $\epsilon_{i}^{\lambda_{u}}$ have finite values if $u$ equals $\mathrm{Zr}$ or $\mathrm{H}$ and tend to infinity if $u=\square$. The interstitial disordered $\mathrm{H} / \square$ interstitial sublattice of the $\mathrm{ZrH}_{c} \square_{2-c}$ system, which will be the reference for the perturbation calculation, is described within the CPA $[16,17]$, which is well suited to account for the strong diagonal disor$\operatorname{der}$ [41] between vacancies and hydrogen atoms. Indeed, this 
approximation acts on only the first term (diagonal elements) of the TB Hamiltonian (1) by replacing interstitial energy levels $\epsilon_{i}^{\lambda_{H}}$ and $\epsilon_{i}^{\lambda_{\square}}=\infty$ by an effective average complex potential $\Sigma$, computed self-consistently through the following iterative equation:

$$
c \tau^{\mathrm{H}}+(2-c) \tau^{\square}=0,
$$

with

$$
\tau^{\mathrm{H}}=\frac{\epsilon^{s_{\mathrm{H}}}-\Sigma(E)}{1-\left[\epsilon^{s_{\mathrm{H}}}-\Sigma(E)\right] \bar{G}_{\mathrm{H}}(E)}, \quad \tau^{\square}=-\frac{1}{\bar{G}_{\mathrm{H}}(E)},
$$

where $\bar{G}_{\mathrm{H}}(E)$ represents the Green's function of the average interstitial medium and depends on $\Sigma(E)$.

Starting from this Hamiltonian representation of interstitial disorder, one can apply the GPM [10-12], whose fundamental idea is that the energy of any ordered structure can be obtained by perturbation expansion from the disordered medium, taken as a reference. The band energy of a given ordered compound $X_{c}$ at concentration $c$ then reads, up to a constant, as follows in terms of $p$-multiplet interactions:

$$
E_{\text {band }}\left(X_{c}\right)=E_{0}(c)+\sum_{p} \sum_{i_{1} i_{2} \cdots i_{p}} \mathscr{C}_{i_{1} i_{2} \cdots i_{p}}^{p}(c),
$$

where $\mathscr{C}_{i_{1} i_{2} \ldots i_{p}}^{p}(c)$ represents the multiplet interaction between $p$ hydrogen atoms located on $i_{1} i_{2} \cdots i_{p}$ neighbor tetrahedral interstitial sites. These multiplet interactions are given by the following analytic formulas:

$$
\begin{aligned}
\mathcal{C}_{i_{1} \cdots i_{p}}^{\mathrm{H} \cdots \mathrm{H}}(c) & =\frac{2}{\pi} \operatorname{Im} \int_{-\infty}^{E_{F}} d E_{1}\left\{\frac{1}{\left\{1-\left[\epsilon^{S \mathrm{H}}-\Sigma\left(E_{1}\right)\right] \bar{G}_{\mathrm{H}}\left(E_{1}\right)\right\} \bar{G}_{\mathrm{H}}\left(E_{1}\right)}\right\}^{p} \\
& \times \frac{1}{\left(N_{\vec{k}}\right)^{p}} \sum_{\vec{k}_{1}, \ldots, \vec{k}_{p}} \operatorname{Tr}\left[G_{i_{1} i_{2}}^{\mathrm{HH}}\left(E_{1}, \vec{k}_{1}\right) \cdot e^{i \vec{k}_{1} \cdot \vec{r}_{1_{2} i_{2}}} G_{i_{2} i_{3}}^{\mathrm{HH}}\left(E_{1}, \vec{k}_{2}\right)\right. \\
& \left.\times e^{i \vec{k}_{2} \cdot \vec{r}_{i_{2} i_{3}}} \ldots G_{i_{p} i_{1}}^{\mathrm{HH}}\left(E_{1}, \vec{k}_{p}\right) \cdot e^{i \vec{k}_{p} \cdot \vec{r}_{i_{i} i_{1}}}\right]
\end{aligned}
$$

where $E_{F}$ is the energy of the Fermi level [given by $N\left(E_{F}\right)=$ $4.0+1.0 c$ for the concentration $c$ under consideration], $N_{\vec{k}}$ is the number of mesh points $\vec{k}$ of the first Brillouin zone, and $G_{i j}^{\mathrm{HH}}\left(E_{1}, \vec{k}\right) \cdot e^{i \vec{k} \cdot \vec{r}_{i j}}$ is the projection at sites $i$ and $j$ on the $\vec{k}$ point of the interstitially disordered medium Green's function $\bar{G}_{\mathrm{H}}(E)$, obtained by solving CPA equation (2).

Thus, if one keeps only the second-order terms of the cluster expansion (truncation to pairwise interactions), the band energy (3) can then be written in the form

$$
\begin{aligned}
E_{\text {band }}\left(X_{c}\right)= & \bar{E}(c)-\left(\frac{c}{2}\right)^{2} \sum_{j} t_{j \text { moy }}^{\mathrm{ZrH}_{2}} V_{j}(c) \\
& +\left(\frac{c}{2}\right) \sum_{j} t_{j \text { moy }}^{\mathrm{X}_{c}} V_{j}(c),
\end{aligned}
$$

where $t_{j \text { moy }}^{\mathrm{X}_{c}}\left(t_{j \text { moy }}^{\mathrm{ZrH}_{2}}\right)$ is the mean number of $\mathrm{H} j$ th neighbors of an atom $\mathrm{H}$ in the ordered structure $X_{c}\left(\mathrm{ZrH}_{2}\right) . V_{j}(c)=\mathscr{C}_{i_{1} i_{2}}^{2}(c)$ is the second-order term from the previous expansion, which represents the pair interactions between two hydrogen atoms located on the $j$ th neighbor tetrahedral interstitial sites. It
TABLE I. Energy levels (eV) and Slater-Koster parameter $\beta(r)=\beta_{i j}^{0} \exp \left[-q_{i j}\left(r-r_{i j}\right) / r_{i j}\right]$ (in eV) for hopping integrals. $r_{i j}$ is the interatomic distance between first neighbors $\left(r_{\mathrm{ZrZr}}=3.20 \AA\right.$, and $\left.r_{\mathrm{ZrH}}=2.09 \AA\right), q_{\mathrm{ZrZr}}=2.40$, and $q_{\mathrm{ZrH}}=1.8$.

\begin{tabular}{lcccr}
\hline \hline orb. i & $\epsilon_{i}$ & coupling & $\beta_{\mathrm{ZrZr}}^{0}$ & $\beta_{\mathrm{ZrH}}^{0}$ \\
\hline $\mathrm{sH}$ & 7.35 & $d d \sigma$ & -1.036 & \\
$\mathrm{sZr}$ & 13.0 & $d d \pi$ & $-0.53 d d \sigma$ & \\
$\mathrm{pZr}$ & 18.0 & $d d \delta$ & $0.10 d d \sigma$ & \\
$\mathrm{dZr}$ & 10.6 & $p d \sigma$ & 1.179 & \\
& & $p d \pi$ & $-0.30 \mathrm{pd \sigma}$ & \\
& & $p p \sigma$ & 2.195 & \\
& & $p p \pi$ & $-0.10 \mathrm{pp \sigma}$ & \\
& & $s d \sigma$ & $0.74 \mathrm{pd \sigma}$ & -1.997 \\
& & $s s \sigma$ & $-0.46 \mathrm{pp \sigma}$ & -1.517 \\
& & $s p \sigma$ & $0.66 \mathrm{pp \sigma}$ & 1.436 \\
\hline \hline
\end{tabular}

reads

$$
\begin{aligned}
V_{j}(c)= & \mathscr{C}_{i_{1} i_{2}}^{2}(c)=-\frac{2}{\pi} \operatorname{Im} \int_{-\infty}^{E_{\mathrm{F}}} d E\left(\tau^{\mathrm{H}}-\tau^{\square}\right)^{2} \frac{1}{N_{\vec{k}} N_{\vec{k}^{\prime}}} \\
& \times \sum_{\vec{k}, \vec{k}^{\prime}}\left\langle i_{1}\left|\bar{G}_{\mathrm{H}}(E, \vec{k}) \bar{G}_{\mathrm{H}}^{\dagger}\left(E, \overrightarrow{k^{\prime}}\right) \cdot e^{i\left(\vec{k}-\overrightarrow{k^{\prime}}\right) \cdot\left(\overrightarrow{r_{1}}-\vec{r}_{i_{2}}\right)}\right| i_{2}\right\rangle .
\end{aligned}
$$

To implement our procedure numerically, one needs a parametrization of the TB Hamiltonian (1). This is performed by extracting both energy levels and hopping integrals by fitting the TB electronic densities of states to DFT ones, first for bulk $\mathrm{Zr}$, then for $\mathrm{ZrH}_{2}$, assuming that direct $\mathrm{H}-\mathrm{H}$ hopping integrals are negligible $[42,43]$. All these parameters are presented in Table I.

In regard to the Hamiltonian representation of the fluoritelike geometry of the $\mathrm{ZrH}_{c} \square_{2-c}$ system (space group $F m \overline{3} m$ ), we have used an $11 \times 11$ TB Hamiltonian matrix (nine $s, p, d$ orbitals for $\mathrm{Zr}$ and two $s$ orbitals for the interstitial medium). Previously used by Papaconstantopoulos et al. [44] to study the electronic structure of $\mathrm{TiH}_{c} \square_{2-c}$ and $\mathrm{VH}_{c} \square_{2-c}$, this formulation splits the simple cubic (sc) interstitial lattice of parameter $a / 2$ into two fcc interstitial lattices of parameter a. We will work with fixed lattice parameter $a=4.82 \AA$, corresponding to that of the stoichiometric phase $\mathrm{ZrH}_{2}$.

\section{PAIR INTERACTION DERIVATION}

\section{A. TB-GPM calculation of multiplet interactions}

We illustrate in Fig. 1 the different multiplet interactions that we consider here, namely, pair interactions $V_{i}$ between $\mathrm{H}$ atoms at $i$-neighbor distance $(i \in\{1, \ldots, 7\})$, triplet interactions $T_{i}$, and quadruplet interactions $Q_{i}$. Regarding the quadruplets, we have selected two paths related to the same cluster but symmetrically nonequivalent and three self-retracing paths, $Q_{6}, Q_{7}$, and $Q_{8}$, which were shown to be predominant in substitutional alloys [12]. Note that two types of interactions exist for third-neighbor pair $V_{3}$ and triplet $T_{7}$ interactions, depending on the presence or not of a $\mathrm{Zr}$ atom in the center of the cluster. In what follows, the calculations will be performed for a mean $V_{3}$ value. 


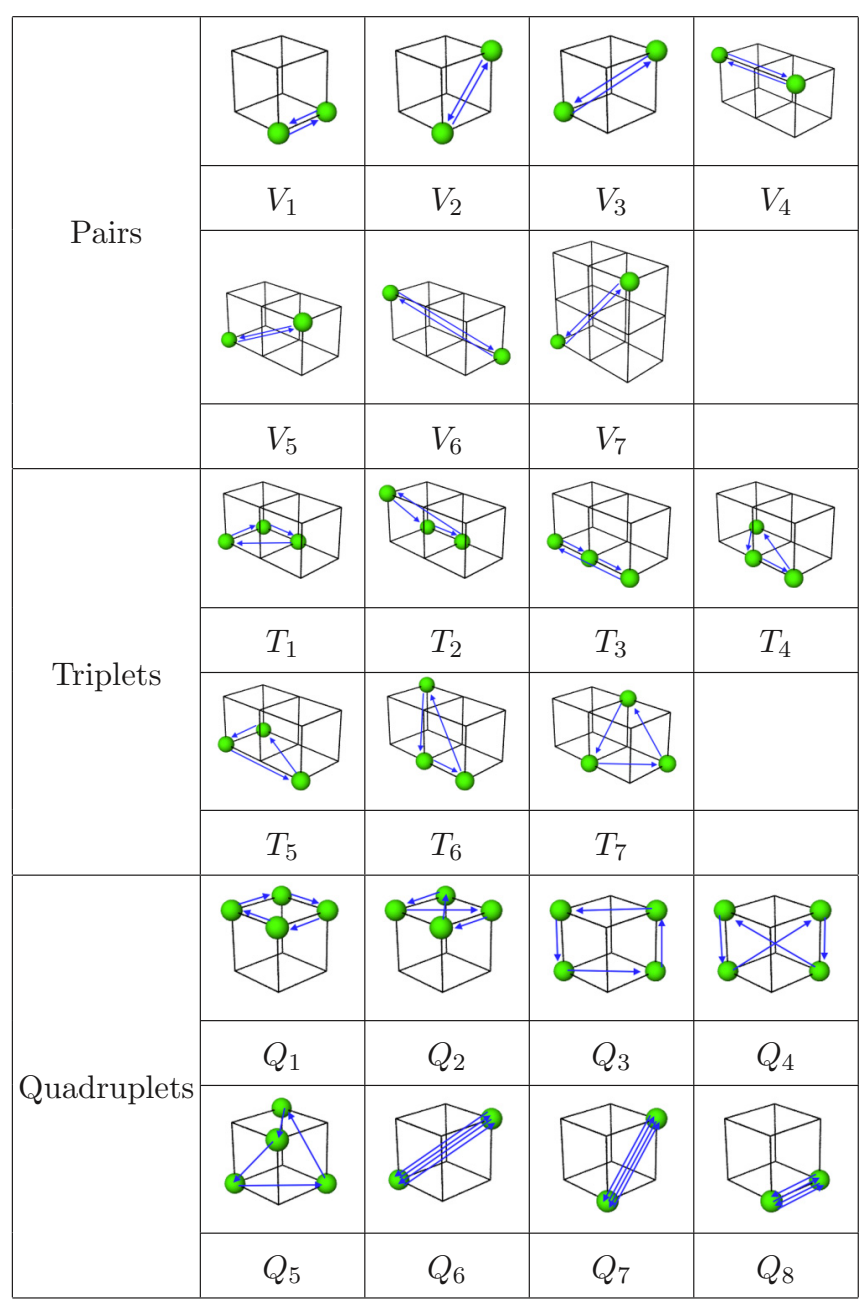

FIG. 1. Multiplet interactions under consideration (only $\mathrm{H}$ atoms on the interstitial SC sublattice are shown).

The corresponding values of these interactions can be seen in Fig. 2 at the concentration $c=1.0$. One can see at first glance that the different energy scales used put in evidence the preponderance of pair interactions $V_{i}$ which are larger

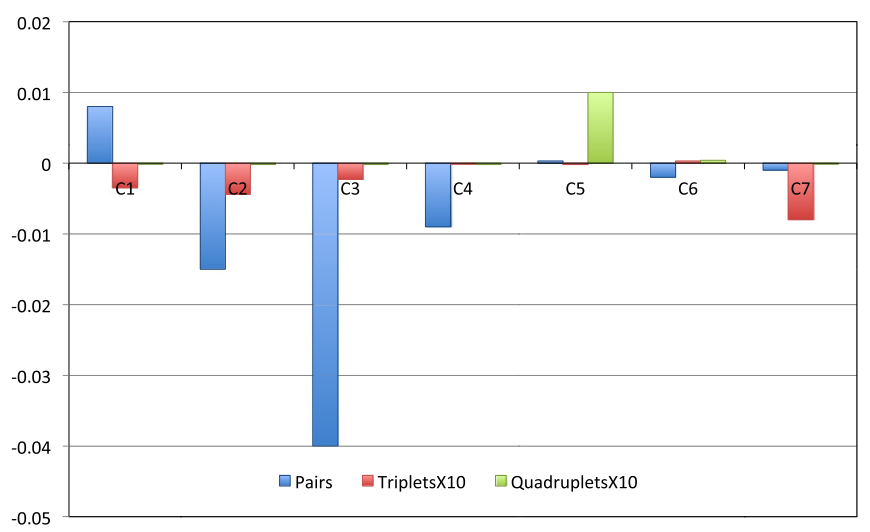

FIG. 2. TB-GPM multiplet interactions (in $\mathrm{eV}$ ) for structures described in Fig. 1. Note the different scales used for the different multiplets. The calculations have been performed for $c=1.0$, with $3280 \vec{k}$ points and 200 points in energy.

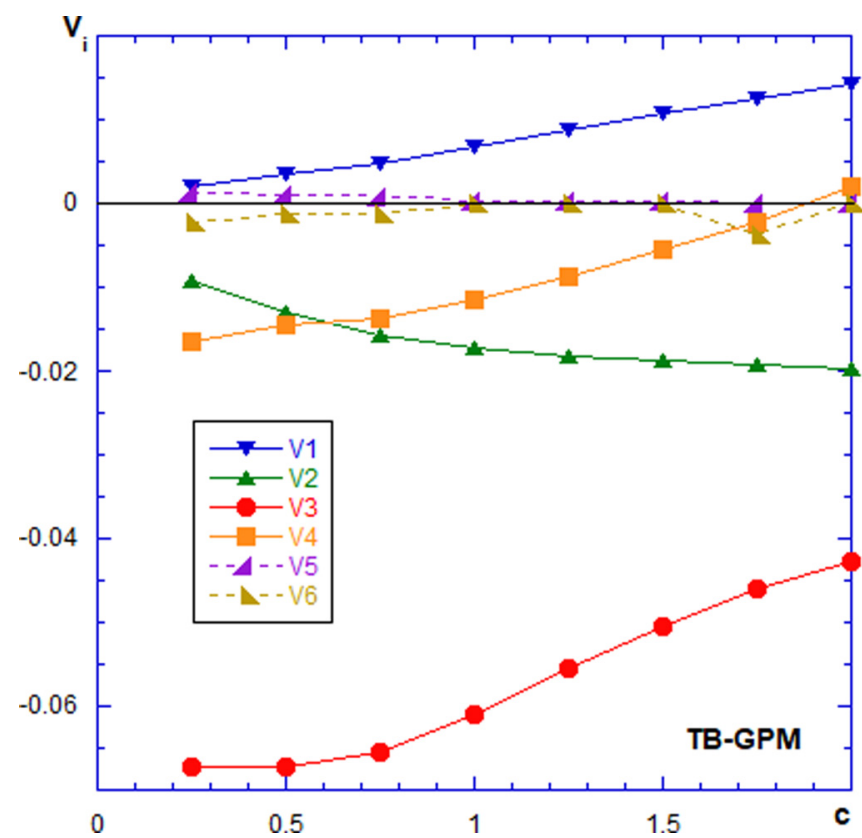

FIG. 3. Hydrogen-hydrogen pairwise interactions $V_{i}(c)(\mathrm{eV})$ as a function of hydrogen concentration $c$.

by an order of magnitude than triplets and by two orders of magnitude than quadruplets, which justifies neglecting them in the following and truncating the cluster development beyond pair interactions.

\section{B. Concentration dependence of TB-GPM effective pair interactions}

Using these implementations in our TB-GPM procedure, one can then apply formula (6) for several concentrations and obtain the curves plotted in Fig. 3, which deserve some comments.

One can first notice that pair potentials $V_{i}(c)$ with $i>4$ are negligible for $c \geqslant 0.5$. Then, the major feature is that configurational energy is dominated (in absolute value) by the third-neighbor interaction $V_{3}$. If one then considers the signs of the interactions, one can see that $V_{1}$ is positive, whereas $V_{2}, V_{3}$, and $V_{4}$ are negative, which means that a hydrogen atom located on an interstitial tetrahedral site tends to be surrounded, on the interstitial lattice, by vacancies in the first neighborhood and hydrogen atoms in the second, third, and fourth neighborhoods.

\section{DFT validation}

The next step is to validate these results through a comparison to more exact DFT calculations. To this aim, we compare energies of ordered structures with the same hydrogen concentration $c$ but different $\mathrm{H} / \square$ occupations for the interstitial tetrahedral sublattice. The sets of ordered structures under considerations for the three concentrations $c=0.5,1.0,1.5$ are presented in Fig. 4. At a given concentration, we perform two comparative calculations of total energy for each structure. First, using $V_{i}(c)$ numerical values and $t_{j \text { moy }}^{\mathbf{X}_{c}}$ factors (obtained by hydrogen-hydrogen neighborhood analysis) in Eq. (5), one gets the band energy, and TB total energy is 


\begin{tabular}{|c|c|c|c|}
\hline & $c=0.5$ & $c=1.0$ & $c=1.5$ \\
\hline $\mathrm{A}_{c}$ & $\begin{array}{r}00 \\
\because 8 \\
0 \\
0\end{array}$ & & $0_{0}^{0}$ \\
\hline $\mathrm{B}_{c}$ & $\begin{array}{c}08 \\
0 \\
0 \\
0 \\
0 \\
0 \\
0\end{array}$ & $0_{0}^{0}$ & 0 \\
\hline $\mathrm{C}_{c}$ & $0_{0}^{0}$ & & $0_{0}^{0.88}$ \\
\hline $\mathrm{D}_{c}$ & & & \\
\hline $\mathrm{E}_{c}$ & & $0_{0}^{0}$ & \\
\hline $\mathrm{F}_{c}$ & & $\frac{0}{0}$ & \\
\hline
\end{tabular}

FIG. 4. Elementary cell $\mathrm{ZrH}_{c} \square_{2-c}$ ordered structures (drawn with OVITO [45] with the color code being blue for $\mathrm{Zr}$, green for $\mathrm{H}$, and red for vacancy), used for TBIM validation.

then deduced by the addition of a repulsive Born-Mayer term $E_{\text {rep }}(c)$ [46] assumed to be fixed at a given concentration $c$ : $E_{\text {tot }}\left(X_{c}\right)=E_{\text {band }}\left(X_{c}\right)+E_{\text {rep }}(c)$. Then we calculate the same total energy using DFT with the VASP code [47-50] for a rigid lattice in the generalized gradient approximation functional.

The corresponding results are compared in Fig. 5, where the energies are referred to that of the stablest structure for each concentration. As can be seen, the energy through TBGPM leads qualitatively to the stability sequence given by DFT calculations. Moreover, in spite of the small values of these stability energy differences, one finds a satisfactory semiquantitative agreement between the TB-GPM and DFT sequences for each composition, with the TB-GPM energies found to be larger by about $15 \%$ for $c=0.5,20 \%$ for $c=1.0$, and about $50 \%$ for $c=1.5$ (note that in the latter case the absolute values are significantly lower).

This agreement validates our perturbation method and definitely confirms that pairwise interactions are sufficient to describe $\mathrm{H} / \square$ sublattice order in zirconium hydrides, the leading ones being those between third neighbors. Finally, let us note that the $\mathrm{D}_{1.0}$ phase, determined as the stablest one at $c=1.0$, is the equivalent of the fct $\gamma$ phase experimentally observed at this concentration $[34,35]$.

\section{Orbital symmetry analysis}

The relative stabilities of ordered systems displayed in Fig. 5 can be explained qualitatively by symmetry considerations. Let us consider the reduced system composed of one $\mathrm{Zr}$ atom and the eight surrounding interstitial sites, occupied by either a hydrogen atom or a vacancy. We focus on the most stable, least stable, and intermediate structures $\mathrm{D}_{1.0}, \mathrm{~A}_{1.0}$, and $\mathrm{F}_{1.0}$, respectively. They belong, respectively, to the symmetry groups $C_{4 v}, T_{d}$, and $D_{2 h}$ and can be described in the $C_{2 v}$ common group framework. The corresponding orbital diagrams obtained by considering a symmetrized linear combination of $s$ orbitals for $\mathrm{H}$ and $s, p, d$ ones for $\mathrm{Zr}$ are illustrated in Fig. 6. We can observe that the threefold-degenerate $\left(t_{2}\right) \mathrm{F}_{1.0}$ highest occupied molecular orbital is partially lifted $\left(e_{2}+a_{1}\right)$ in the $\mathrm{A}_{1.0}$ structure and is fully lifted in the $\mathrm{D}_{1.0}$ one. According to Hund's rule, the $A_{1.0}$ energy will then be higher than the $F_{1.0}$ one, with the latter being higher than the $\mathrm{D}_{1.0}$ one.

Note that this effect holds even though we have simplified the explanation, not considering here the orbital splitting in the TB Hamiltonian in view of the small difference between the $e_{g}$ and $t_{2 g}$ levels. Indeed, this simplification does not change our argument since it plays only a minor role in the relative position of the $1 a_{1}$ energy level (involving the $e_{g} d_{z^{2}} \mathrm{Zr}$ orbitals) and the other three lower orbitals, from $t_{2}$ (involving $t_{2 g} d_{z x}, d_{z y}$, and $d_{x^{2}-y^{2}} \mathrm{Zr}$ orbitals). The major effect is the hydrogen-stabilizing interaction. The $e_{g} d_{x y} \mathrm{Zr}$ orbital is not illustrated here since it is not involved in $\mathrm{H}$ bonding and then presents a higher-energy level.

One can also observe that the $p$ orbitals play a major role in the stabilization of the different structures, which implies that they cannot be neglected. Furthermore, with the symmetry playing a central role in the structure stabilization, one can expect that any deformation, in particular, tetragonalization, could impact relative stabilities. Last, it can also be observed in Fig. 6 that only the $D_{1.0}$ structure exhibits $V_{3}$ interactions, which will induce delocalization between surroundings $\mathrm{Zr}$ tetrahedrons. These trends are perfectly consistent with both the $V_{i}$ relative importance and structure stabilities derived from the presented methodology.

\section{E. Application to cluster expansion derivation of multiplet interactions from DFT calculations}

The first conclusion of the TB-GPM is that the cluster expansion can be truncated to the second-order terms (pair interactions) and that higher-order terms can be neglected. The second conclusion is that, among these pair interactions, only those between first, second, and third (and possibly fourth) neighbors can be taken into account. This allows us an alternative way to derive these pair interactions from DFT calculations by using a reverse method. In practice, we first calculate through DFT the formation energy of different ordered phases, defined as the energy balance between the ordered phase and the two separated $\mathrm{ZrH}_{2}$ and $\mathrm{Zr}$ phases. Then we develop this energy as a function of $V_{1}, V_{2}, V_{3}$, and 


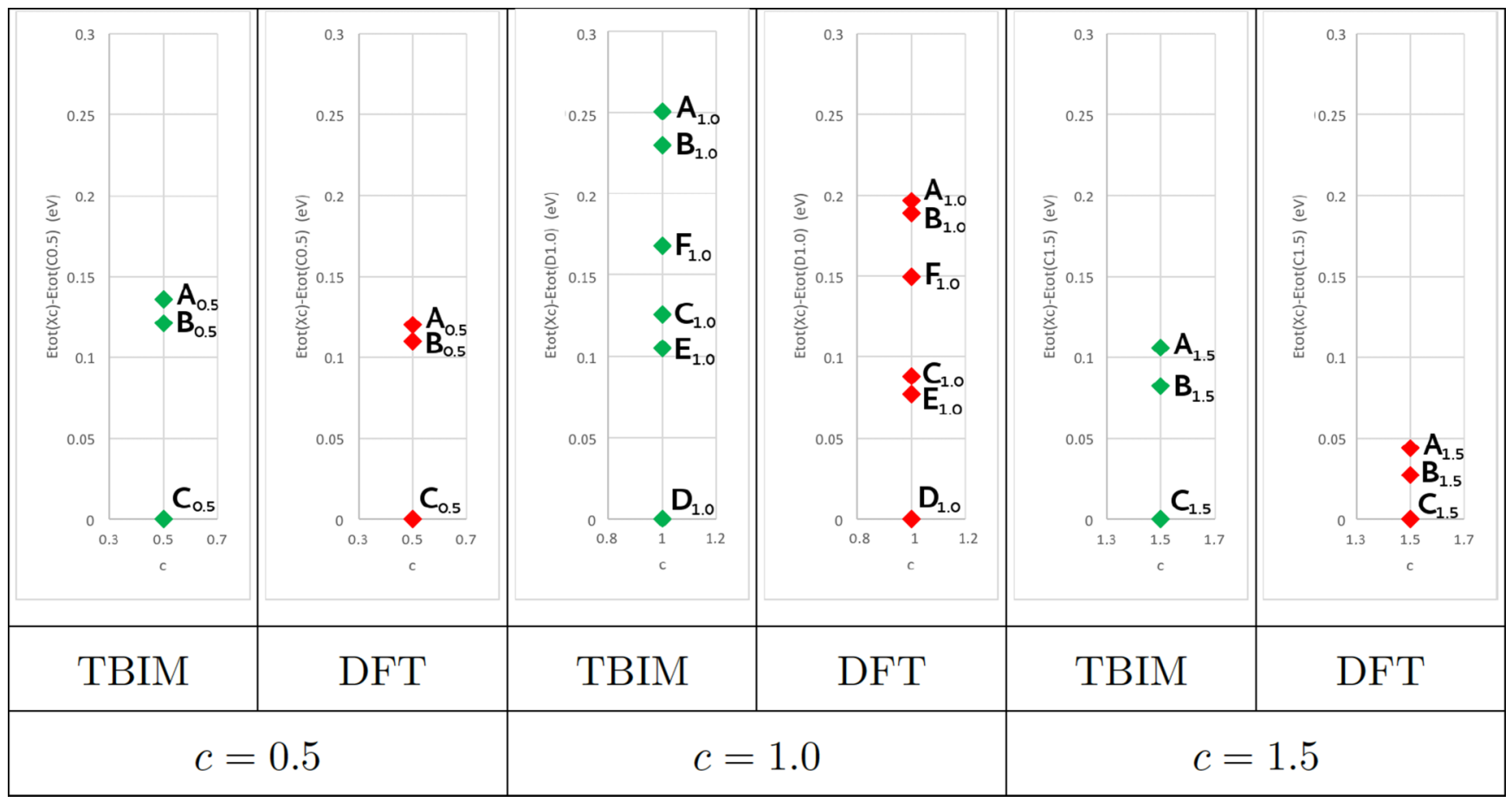

FIG. 5. Comparison between total energy sequences of structures represented in Fig. 4, in TBIM and DFT (VASP).

$V_{4}$, which leads to a set of equations to be solved. To do that, we have added to the previously selected set of ordered structures $(\mathrm{A}, \mathrm{B}, \mathrm{C}, \ldots)$ new structures in order to have a larger number of values to fit (about 12 ordered phases for each concentrations). The resolution of the system then gives the values of the effective pair interactions. Note that taking into account or not $V_{4}$ in the system of equations does not change the values found for $V_{1}, V_{2}$, and $V_{3}$. The curves corresponding to the latter interactions are plotted as dashed lines in Fig. 7.

In addition, the DFT $V_{n}$ curves can be extended to both dilute limits, respectively $c \rightarrow 0$ and $c \rightarrow 2$, by calculating

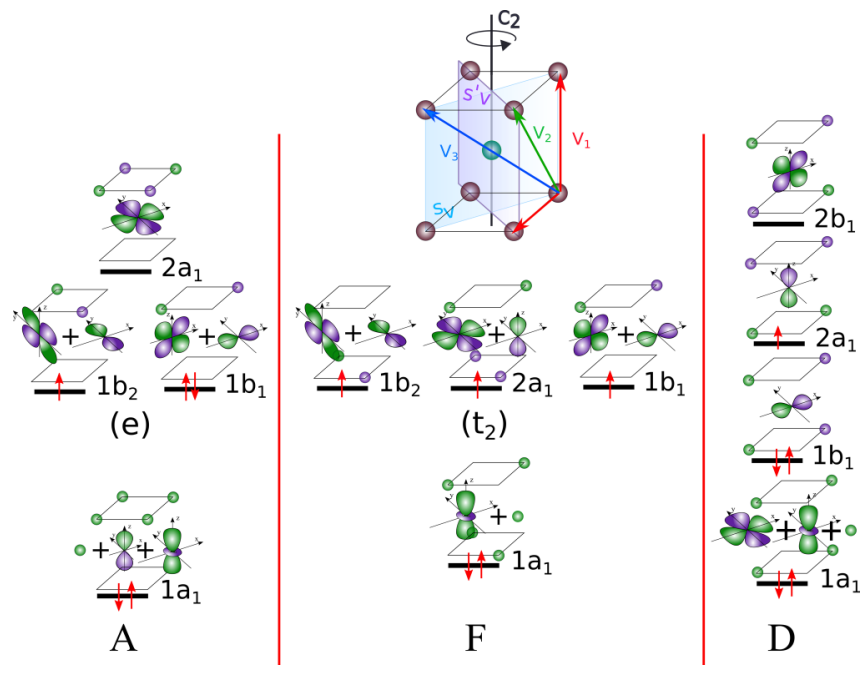

FIG. 6. Walsh diagram of $\mathrm{ZrH}$ tetrahedrons for the $\mathrm{A}_{1.0}, \mathrm{~F}_{1.0}$, and $\mathrm{D}_{1.0}$ structures. The relevant $C_{2 v}$ group of symmetries is illustrated at the top. Only the lower occupied levels are illustrated. the same interactions $V_{n}$ now directly from bringing respectively two $\mathrm{H}$ impurities in pure $\mathrm{Zr}$ bulk and two vacancies in the $\mathrm{ZrH}_{2}$ compound. These pairs of impurities are taken first in $n$ th-neighbor positions, then as isolated impurities, using

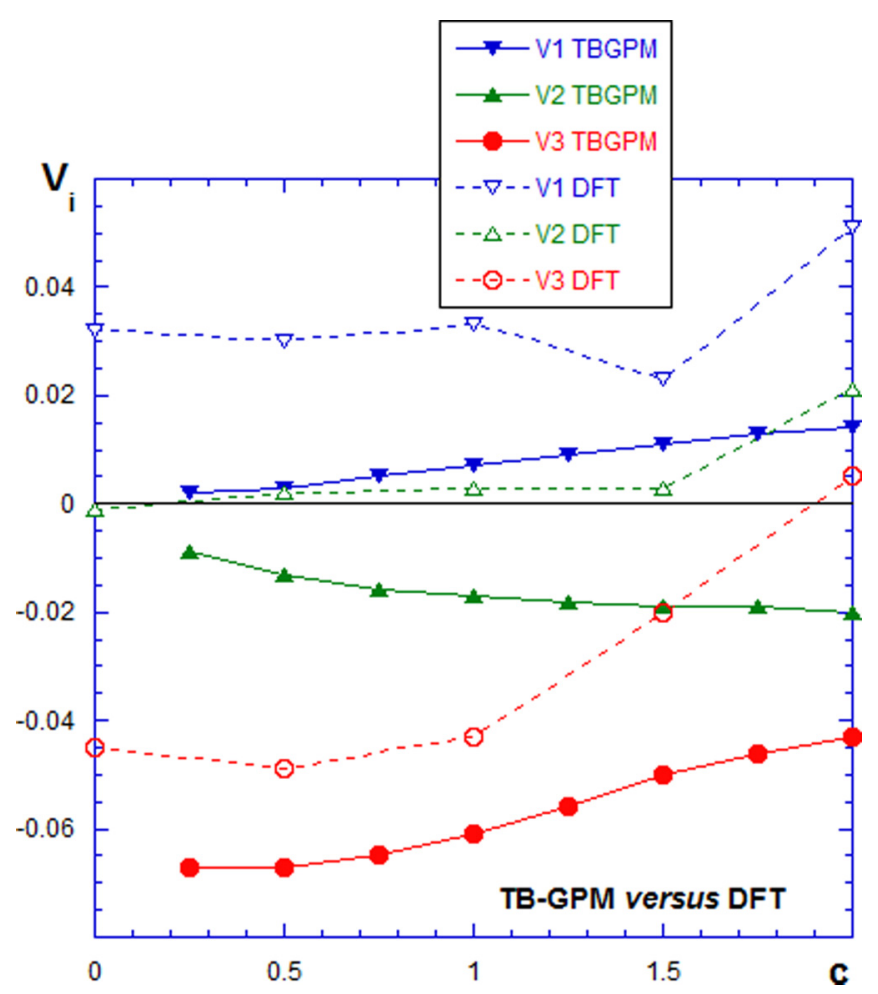

FIG. 7. Hydrogen-hydrogen pairwise interactions $V_{i}(c)(\mathrm{eV})$ issued by structure reverse method from DFT calculations compared to those calculated directly through TB-GPM. 

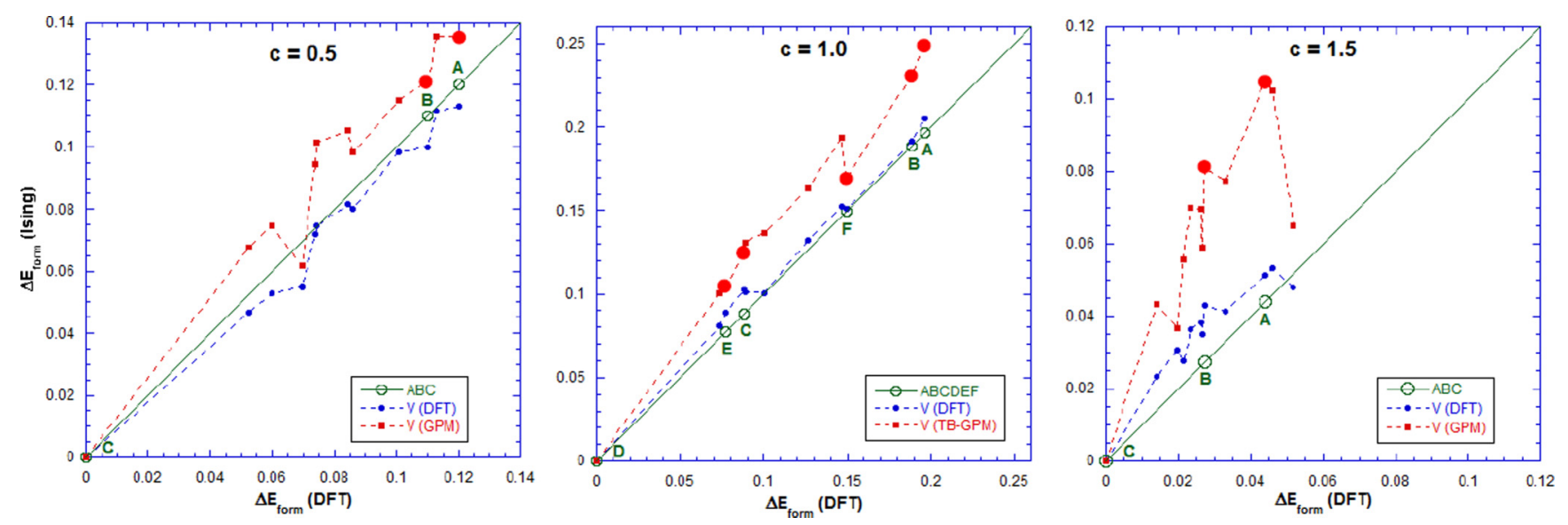

FIG. 8. Formation energies of the various structures relative to the stablest one, $\Delta E_{\text {form }}$, reconstructed with effective pair interactions either calculated directly within TB-GPM (red dashed line) or derived from DFT total energies (blue dashed line) as a function of the actual DFT calculations. The small solid red and blue symbols refer to the different ordered structures used in the fitting procedure. The large solid red (open green) dots recall the TB-GPM calculations (exact DFT values) for the previous limited set of structures A, B, C, ... represented in Fig. 4.

a reasonably large supercell (to avoid spurious interactions due to translational symmetry) and choosing a lattice constant of $4.82 \AA$, like in the case of GPM calculations. The energy balance between both configurations then gives directly the effective pair interaction $V_{n}$ [51]. As can be seen in Fig. 7, the pair interaction values obtained in the dilute limits are perfectly consistent with those derived from DFT energy calculations in concentrated compounds through the reverse method.

One can then compare DFT pair interactions to those directly issued from TB-GPM calculations. This can be achieved by comparing dashed lines with open symbols to solid lines with solid symbols in Fig. 7. The first observation is that the overall trends followed by the different $V_{n}$ as a function of concentration are the same, with the GPM values being shifted by about $20 \mathrm{meV}$ towards smaller energies with respect to DFT ones. This means that the sequence $\left(V_{n}-V_{1}\right)$ is found to be almost identical from both DFT and GPM calculations.

It is worth noticing that the TB-GPM interactions account for only the chemical-induced contribution to the interstitialinterstitial interactions, which amounts to neglecting the strain-induced one which has been shown to be significant in the case of oxygen and nitrogen ordering [30]. However, we have checked that this strain effect is much weaker for a small hydrogen interstitial by allowing atomic displacements in DFT calculations. Indeed, we found that such a strain relaxation leads to only very small modifications of both the atomic environment of the interstitial atoms and the energy balance involved in the effective pair interactions calculations.

Let us also mention that concentration-independent cluster expansion is not well defined if interactions in the system are concentration dependent [52]. In this case, any composition variation may easily destroy cluster expansion if it leads to a considerable change in the electronic structure, which obviously cannot be reproduced whatever the cluster expansion methodology.
One can then wonder to what extent this rigid shift between TB-GPM and DFT effective pair interactions could affect the ability of TB-GPM to account for the respective stabilities of the different ordered structures. To this aim, we plot in Fig. 8 the formation energies relative to the lowest one, denoted $\Delta E_{\text {form }}$, calculated from GPM (red dashed line) as a function of the actual DFT energy values (green diagonal solid line). As can be seen, the TB-GPM values follow the same trend as those issued from DFT total energy calculations (green solid line), consistent with our previous results on the short limited sequence of $\mathrm{ABC} \cdots$ structures (recalled in Fig. 8). More precisely, one finds that $\Delta E_{\text {form }}(\mathrm{GPM}) \simeq \alpha \Delta E_{\text {form }}$ (DFT), with $\alpha=1.15(c=0.5), 1.2(c=1.0), 2(c=1.5)$. For the sake of comparison we also plot in Fig. 8 the formation energies calculated with the DFT pair interactions with respect to the minimal one for all the structures involved in the fit. As can be seen, the agreement with exact DFT values is satisfactory, although not perfect, as it would be for the sequence of formation energies which have, indeed, been fitted.

\section{APPLICATIONS}

\section{A. Phase diagram of the $\mathrm{ZrH}_{c} \square_{2-c}$ system}

One can introduce temperature for a preliminary thermostatistical exploration of H-vacancy interstitial ordering on the tetrahedral interstitial sublattice through a standard canonical Monte Carlo approach. To this aim, we employ here the $\mathrm{H}-\mathrm{H}$ pairwise interaction model, derived by CEM from DFT calculations in Sec. III E. Our study is obviously restricted to the tetrahedral interstitial sublattice of the fcc $\mathrm{ZrH}_{c} \square_{2-c}$ system at fixed lattice parameter $a=4.82 \AA$ : In other words, that is equivalent to an investigation of the finite temperature ordering processes of an $\mathrm{H}$-vacancy binary alloy on an sc rigid lattice. To perform Monte Carlo simulations, we have used several simulation boxes containing 8000 tetrahedral interstitial sites, with respective hydrogen concentrations of $0.250,0.500,0.750,1.000,1.125,1.250,1.375,1.500,1.625$, 1.750, and 1.875. Energy was computed using Eq. (5), with 


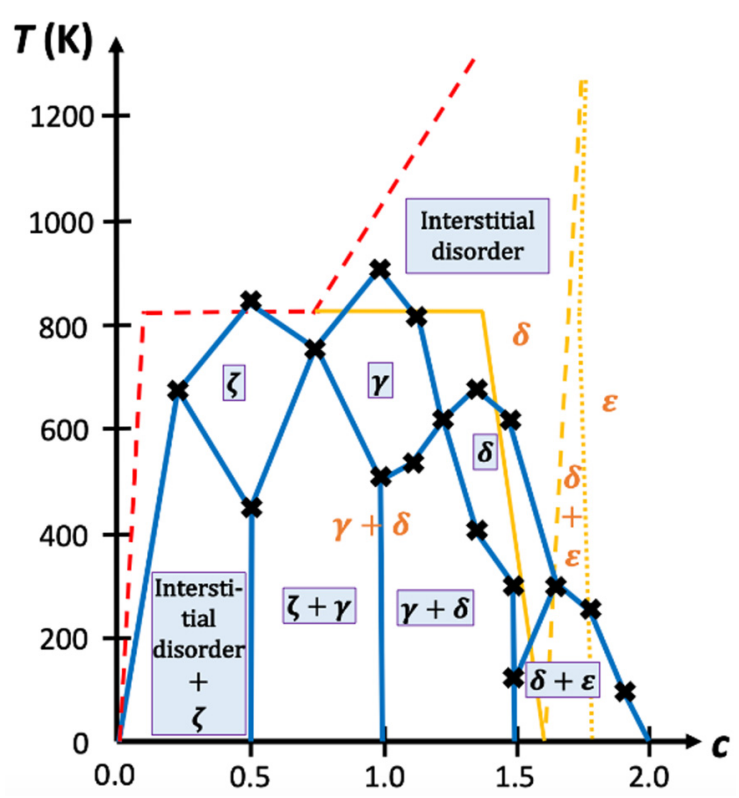

FIG. 9. Phase diagram of the $\mathrm{ZrH}_{c} \square_{2-c}$ system obtained by Monte Carlo simulation, compared with experimental data [53]. The Monte Carlo results are displayed by blue solid lines and black symbols; the experimental ones are displayed by red and yellow lines. The red line shows the limit of fcc-fct hydride existence domains.

pairwise interactions $V_{i}(c)$ fitted to values derived by CEM at $4.82 \AA$. For each simulation box, we performed a heating up to $1400 \mathrm{~K}$ in steps of $20 \mathrm{~K}$ with $810^{6}$ Metropolis iterations at a given temperature. Ordering phenomena were followed by studying the evolution of hydrogen-hydrogen neighbors up to third order. The results thus obtained are summarized in Fig. 9, where they are compared to experimental data. In order to make this direct comparison possible, we have replaced our theoretical notations with their experimental counterparts, namely, $\zeta, \gamma, \delta$, and $\epsilon$ phases for the $\mathrm{C}_{0.5}, \mathrm{D}_{1.0}, \mathrm{C}_{1.5}$ and $\mathrm{ZrH}_{2}$ structures, respectively.

As can be seen, in the pertinent range of concentration of our study $(1.0 \leqslant c \leqslant 2.0$, corresponding to the experimental stability domain of the fcc and fct phases), the calculations reproduce quite well the stability domains of the $\gamma$ and $\delta$ phases and coexistence domains $(\gamma+\delta)$ and $(\delta+\epsilon)$, consistent with experimental phase boundaries [53]. In addition, note that even at lower concentrations, recent DFT calculations [54] have confirmed the stability of the $\zeta$ phase built on the fcc lattice, as predicted by our calculations, at $c=0.5$. To go further and take into account distortions of the fcc lattice, one will need to introduce stress dependence for pairwise interactions.

\section{B. Stress impact}

Let us illustrate the potential application of this description when the external variation is due to stress. Indeed, up to now all the calculations have been performed at fixed lattice parameter $a=4.82 \AA$. We then studied the impact of an isotropic deformation of the lattice on the effective pair interactions, through the lattice parameter exponential

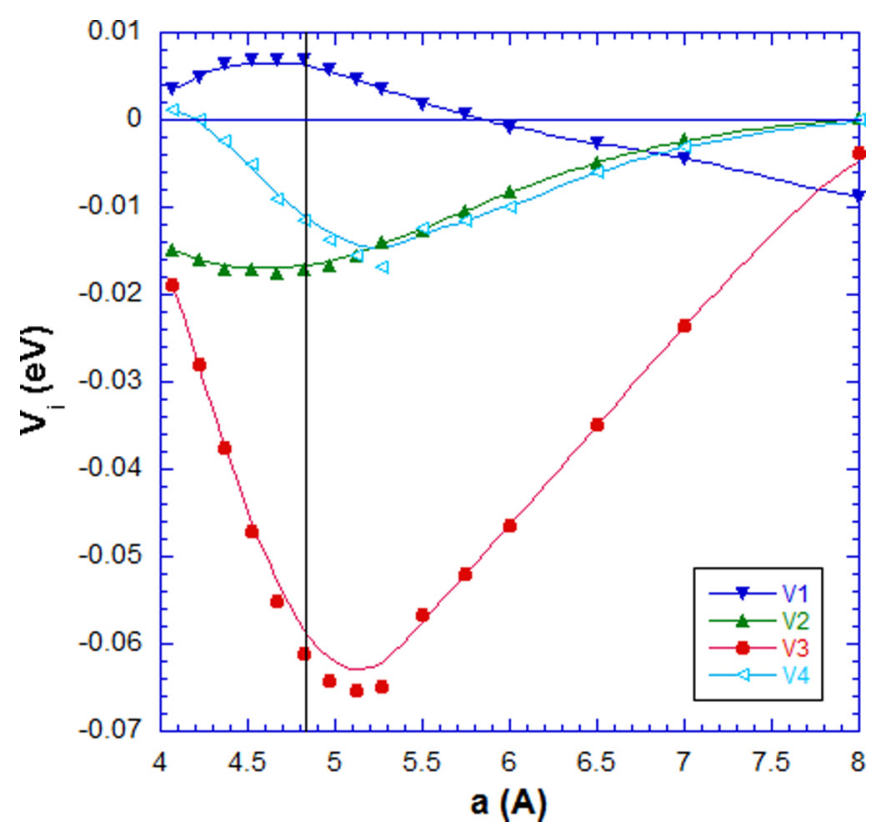

FIG. 10. Stress effect on ordering for $c=1.0$ : variation of $V_{i}$ as a function of the lattice parameter from TB-GPM calculations.

dependence of hopping integrals in Hamiltonian (1). This is illustrated at $c=1$ in Fig. 10. As can be seen, globally, all the interactions present an extremum around the value of the equilibrium lattice parameter and decrease (in absolute value) for both dilation and compression. This decrease is more abrupt on the compressive side. In spite of these variations, the $V_{3}$ interaction remains the leading interaction whatever the deformation. Such behavior does not change the $c=0.5$ and $c=1.5$ sequences but tends to a stabilization of $\mathrm{F}_{1.0}$ at $c=1.0$. This observation can be followed by the variation of the formation energies of the different ordered structures as a function of the lattice parameter, plotted in Fig. 11 (left-hand side).

In order to confirm the ability of our model to account for the stress effect on ordering, we have once again performed DFT calculations to determine the evolution of the total energy as a function of lattice deformation, in the case $c=1$. This leads to the results plotted in Fig. 11 (right-hand side), in which we draw the variation of the stability energies of the different ordered phases either reconstructed from TB-GPM pair interactions or directly calculated from DFT calculations. As can be seen in Fig. 11, the DFT calculations confirm semiquantitatively the main results of TB-GPM calculations:

(i) The overall stability sequences remain unchanged whatever the deformation.

(ii) The $\mathrm{D}_{1.0}$ structure remains the most stable one whatever the deformation except for the very strong compressions which stabilize the $F_{1.0}$ one (in particular in the TB-GPM description).

(iii) The overall behavior as a function of deformation is the same, passing through a maximum (which is shifted by $1 \AA$ between both approaches) with a low decrease on the dilation side and a sharp one on the compressive side. 

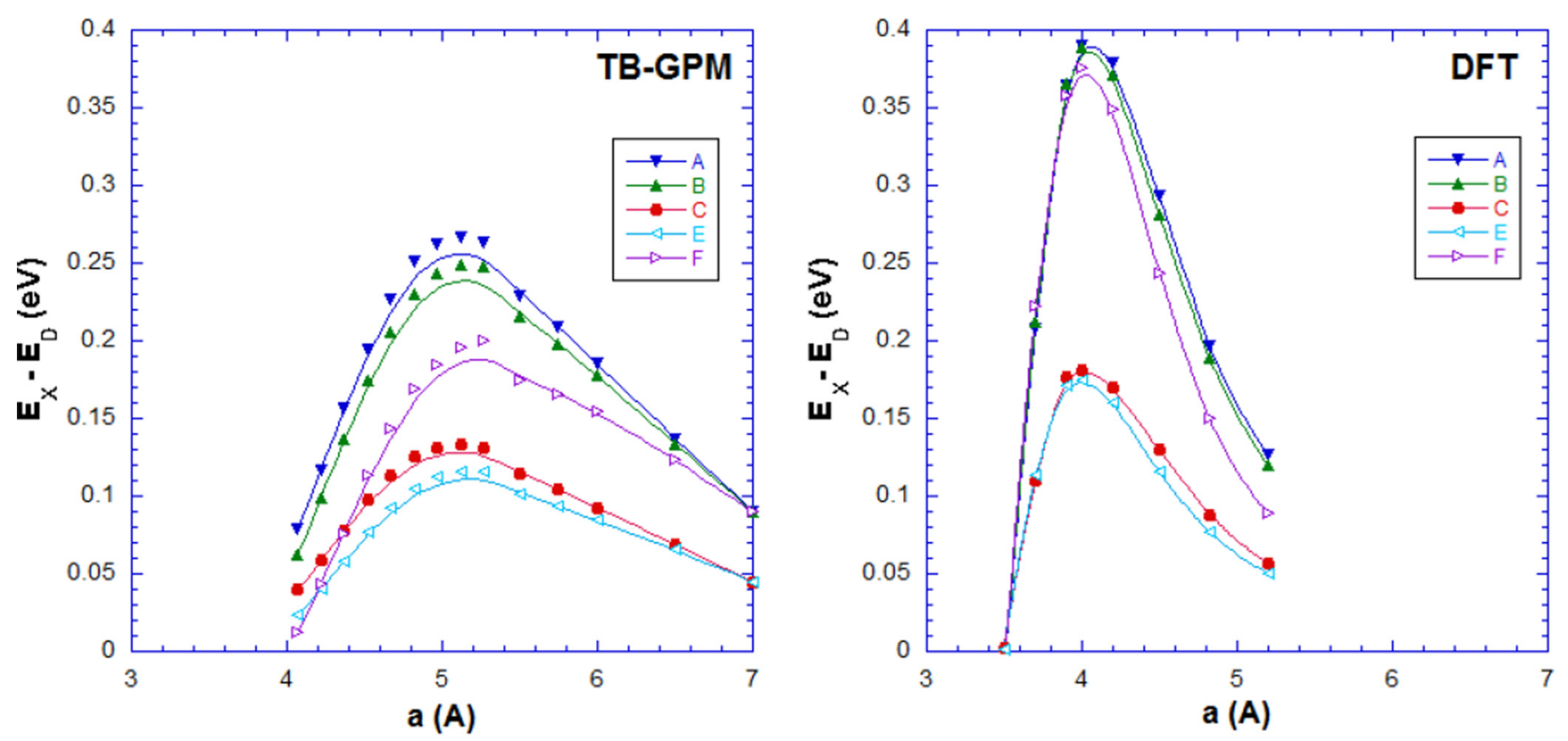

FIG. 11. Variation of the formation energies of the $\mathrm{A}_{1.0}, \mathrm{~B}_{1.0}, \mathrm{C}_{1.0}, \mathrm{E}_{1.0}$, and $\mathrm{F}_{1.0}$ structures referred to the $\mathrm{D}_{1.0}$ one as a function of the lattice parameter, either reconstructed from the TB-GPM pair interactions of Fig. 10 (left) or directly calculated through DFT (right).

\section{CONCLUSION}

To summarize, we have presented here a TB-GPM method which allows us to calculate directly the cluster interactions involved in the configurational energy of metal hydrides and to characterize their concentration and stress dependence. Such a method therefore gives a useful guide to identify the relevant cluster interactions (leading terms, range) to take into account in the widely used cluster expansion method to derive interactions from DFT total energy calculations in order to study ordering processes in interstitial metallic hydrides through appropriate thermostatistical simulations. In the particular case of the $\mathrm{Zr}-\mathrm{H}$ system, one finds that (i) multiplet interactions can be neglected beyond pair interactions, (ii) these pair interactions are negligible beyond fourth $\mathrm{H}-\mathrm{H}$ neighbors, (iii) the predominant interaction is that between third neighbors $V_{3}$, and (iv) a hydrogen atom tends to be surrounded by vacancies in the first-order neighborhood and hydrogen atoms in the second, third, and fourth neighborhoods. These results have been quantitatively confirmed by comparison to DFT calculations and qualitatively interpreted through orbital symmetry arguments. This sodetermined hierarchy of multiplet interactions gave us an alternative way to derive these pair interactions from DFT calculations by using a cluster expansion method. These DFT-CEM pair interactions were then used in Monte Carlo simulations to determine a $\mathrm{ZrH}$ phase diagram which is found to be in quite good agreement with experimental observations. Finally, we have characterized the effect of isotropic stress on the TB-GPM pair interactions, which revealed an impact focused on $V_{3}$, leading to a possible structural transition.

\section{ACKNOWLEDGMENTS}

The authors would like to thank the French Agence Nationale de la Recherche (SIZHYP ANR-12-BS09-0029 project) for financial support.
[1] J. M. Sanchez, F. Ducastelle, and D. Gratias, Generalized cluster description of multicomponent systems, Physica A (Amsterdam, Neth.) 128, 334 (1984).

[2] J. W. D. Connolly and A. R. Williams, Density functional theory applied to phase transformations in transition metal alloys, Phys. Rev. B 27, 5169 (1983).

[3] L. Schlapbach and A. Züttel, Hydrogen-storage materials for mobile applications, in Materials for Sustainable Energy: A Collection of Peer-Reviewed Research and Review Articles from Nature Publishing Group (World Scientific, Singapore, 2011), p. 265.

[4] W. M. Mueller, J. P. Blackledge, and G. G. Libowitz, Metal Hydrides (Academic Press, New York, 2013).
[5] S. C. Lumley, R. W. Grimes, S. T. Murphy, P. A. Burr, A. Chroneos, P. R. Chard-Tuckey, and M. R. Wenman, The thermodynamics of hydride precipitation: The importance of entropy, enthalpy and disorder, Acta Mater. 79, 351 (2014).

[6] A. Van der Ven, Q. Xu, J. Bhattacharya, in First-principles prediction of hydride phase stability and the thermodynamics of decohesion, edited by B. Somerday, P. Sofronis, and R. H. Jones (ASM International, Ohio, 2009), pp. 632-639.

[7] L. Holliger, A. Legris, and R. Besson, Hexagonal-based ordered phases in H-Zr, Phys. Rev. B 80, 094111 (2009).

[8] R. Besson and R. Candela, Ab initio thermodynamics of fcc H$\mathrm{Zr}$ and formation of hydrides, Comput. Mater. Sci. 114, 254 (2016). 
[9] T. C. Kerscher, G. Schöllhammer, P. Herzig, W. Wolf, R. Podloucky, and S. Müller, First-principles study of hydrogen ordering in lanthanum hydride and its effect on the metalinsulator transition, Phys. Rev. B 86, 014107 (2012).

[10] F. Gautier, F. Ducastelle, and J. Giner, Ordering and segregation processes in transition metal alloys in relation to their electronic structures, Philos. Mag. 31, 1373 (1975).

[11] F. Ducastelle and F. Gautier, Generalized perturbation theory in disordered transitional alloys: Applications to the calculation of ordering energies, J. Phys. F 6, 2039 (1976).

[12] A. Bieber and F. Gautier, Multiatom interactions, order and stability in binary transition metal alloys, Z. Phys. B 57, 335 (1984)

[13] G. Tréglia, B. Legrand, and F. Ducastelle, Segregation and ordering at surfaces of transition metal alloys: The tight-binding Ising model, Europhys. Lett. 7, 575 (1988).

[14] G. Tréglia, F. Ducastelle, and F. Gautier, Generalised perturbation theory in disordered transition metal alloys: Application to the self-consistent calculation of ordering energies, J. Phys. F 8, 1437 (1978).

[15] C. M. Goringe, D. R. Bowler, and E. Hernandez, Tight-binding modeling of materials, Rep. Prog. Phys. 60, 1447 (1997).

[16] P. Soven, Coherent-potential model of substitutional disordered alloys, Phys. Rev. 156, 809 (1967).

[17] J. S. Faulkner, Electronic states of substoichiometric compounds and application to palladium hydride, Phys. Rev. B 13, 2391 (1976).

[18] J. P. Landesman, G. Tréglia, P. Turchi, and F. Ducastelle, Electronic structure and pairwise interactions in substoichiometric transition metal carbides and nitrides, J. Phys. (Paris) 46, 1001 (1985).

[19] J. P. Julien, P. E. A. Turchi, and D. Mayou, Real-space solution of the coherent potential approximation equations in the Shiba approximation, Phys. Rev. B 64, 195119 (2001).

[20] P. E. A. Turchi, D. Mayou, and J. P. Julien, Real-space tightbinding approach to stability and order in substitutional multicomponent alloys, Phys. Rev. B 56, 1726 (1997).

[21] P. E. A. Turchi and D. Mayou, Real-space electronic structure approach to transport in alloys, Phys. Rev. B 64, 075113 (2001).

[22] D. Mayou, P. E. A. Turchi, S. Roche, and J. P. Julien, Electronic structure and transport in non-periodic systems: New $\mathrm{O}(\mathrm{N})$ methods, in Proceedings of the Fall MRS Meeting on "TightBinding Approach to Computational Materials Science," edited by by P. E. A. Turchi, A. Gonis, and L. Colombo, MRS Online Proceedings Library Archive Vol. 491 (Cambridge University Press, England, 1998), p. 231.

[23] P. E. A. Turchi, D. Mayou and J. P. Julien, Real-space tightbinding approach to order in complex alloys, in Proceedings of the 9th International Conference on Modern Materials and Technologies (CIMTEC'98), edited by P. Vincenzini (Techna Publishers, Faenza, Italy, 1998), p. 83.

[24] P. E. A. Turchi, G. M. Stocks, W. H. Butler, D. M. Nicholson, and A. Gonis, First-principles study of ordering properties of substitutional alloys using the generalized perturbation method, Phys. Rev. B 37, 5982 (1988).

[25] P. E. A. Turchi, A. Gonis, V. Drchal, and J. Kudrnovský, Firstprinciples study of stability and local order in substitutional TaW alloys, Phys. Rev. B 64, 085112 (2001).
[26] P. E. A. Turchi, M. Sluiter, F. J. Pinski, D. D. Johnson, D. M. Nicholson, G. M. Stocks, and J. B. Staunton, First-Principles Study of Phase Stability in Cu-Zn Substitutional Alloys, Phys. Rev. Lett. 67, 1779 (1991); Erratum: First-Principles Study of Phase Stability in Cu-Zn Substitutional Alloys, 68, 418 (1992).

[27] A. V. Ruban, S. Shallcross, S. I. Simak, and H. L. Skriver, Atomic and magnetic configurational energetics by the generalized perturbation method, Phys. Rev. B 70, 125115 (2004).

[28] P. A. Korzhavyi, L. V. Pourovskii, H. W. Hugosson, A. V. Ruban, and B. Johansson, Ab Initio Study of Phase Equilibria in $\mathrm{TiC}_{x}$, Phys. Rev. Lett. 88, 015505 (2002).

[29] B. Alling, A. V. Ruban, A. Karimi, L. Hultman, and I. A. Abrikosov, Unified cluster expansion method applied to the configurational thermodynamics of cubic $\mathrm{Ti}_{1-x} \mathrm{Al}_{x} \mathrm{~N}$, Phys. Rev. B 83, 104203 (2011).

[30] A. V. Ruban, V. I. Baykov, B. Johansson, V. V. Dmitriev, and M. S. Blanter, Oxygen and nitrogen interstitial ordering in hep Ti, Zr, and Hf: An ab initio study, Phys. Rev. B 82, 134110 (2010).

[31] J. B. Bai, C. Prioul, and D. Francois, Hydride embrittlement in zircaloy-4 plate: Part I. Influence of microstructure on the hydride embrittlement in ZIRCALOY-4 at $20 \mathrm{c}$ and $350 \mathrm{c}$, Metall. Mater. Trans. A 25, 1185 (1994).

[32] J. B. Bai, N. Ji, D. Gilbon, C. Prioul, and D. Francois, Hydride embrittlement in ZIRCALOY-4 plate: Part II. Interaction between the tensile stress and the hydride morphology, Metall. Mater. Trans. A 25, 1199 (1994).

[33] A. T. Motta and L.-Q. Chen, Hydride formation in zirconium alloys, JOM 64, 1403 (2012).

[34] E. Zuzek, J. P. Abriata, A. San-Martin, and F. D. Manchester, The H-Zr (hydrogen-zirconium) system, Bull. Alloy Phase Diagrams 11, 385 (1990).

[35] S. Suman, M. K. Khan, M. Pathak, R. N. Singh, and J. K. Chakravartty, Hydrogen in zircaloy: Mechanism and its impacts, Int. J. Hydrogen Energy 40, 5976 (2015).

[36] C. Domain, R. Besson, and A. Legris, Atomic-scale Ab-initio study of the Zr-H system: I. Bulk properties, Acta Mater. 50, 3513 (2002).

[37] Y. Zhang, C. Jiang, and X. Bai, Anisotropic hydrogen diffusion in $\alpha-\mathrm{Zr}$ and Zircaloy predicted by accelerated kinetic Monte Carlo simulations, Sci. Rep. 7, 41033 (2017).

[38] P. A. Burr, S. T. Murphy, S. C. Lumley, M. R. Wenman, and R. W. Grimes, Hydrogen accommodation in $\mathrm{Zr}$ second phase particles: Implications for $\mathrm{H}$ pick-up and hydriding of Zircaloy2 and Zircaloy-4, Corros. Sci. 69, 1 (2013).

[39] Y. Udagawa, M. Yamaguchi, H. Abe, N. Sekimura, and T. Fuketa, $A b$ initio study on plane defects in zirconiumhydrogen solid solution and zirconium hydride, Acta Mater. 58, 3927 (2010).

[40] G. J. Ackland, Embrittlement and the Bistable Crystal Structure of Zirconium Hydrides, Phys. Rev. Lett. 80, 2233 (1998).

[41] G. Tréglia, C. Goyhenex, C. Mottet, B. Legrand, and F. Ducastelle, Electronic structure of nanoalloys: A guide of useful concepts and tools, In Nanoalloys (Springer, London, 2012), pp. 159-195.

[42] A. Dufresne, G. Tréglia, and F. Ribeiro, Tight-binding nmoment potential for zirconium hydride atomistic modeling, Metall. Res. Technol. 112, 102 (2015). 
[43] A. Dufresne, F. Ribeiro, and G. Tréglia, How to derive tightbinding spd potentials? Application to zirconium, J. Phys.: Condens. Matter 27, 336301 (2015).

[44] D. A. Papaconstantopoulos, P. M. Laufer, and A. C. Switendick, Theory of Electronic States in disordered alloy hydrides, in Hydrogen in Disordered and Amorphous Solids, edited by G. Bambakidis and R. C. Bowman, NATO Advanced Studies Institute, Series B: Physics Vol. 136 (Springer Science+Business Media, New York, 1986), pp. 139-152.

[45] A. Stukowski, Visualization and analysis of atomistic simulation data with ovito-the open visualization tool, Modell. Simul. Mater. Sci. Eng. 18, 015012 (2009).

[46] F. Ducastelle, Modules élastiques des métaux de transition, J. Phys. (Paris) 31, 1055 (1970).

[47] G. Kresse and J. Hafner, Ab initio molecular dynamics for liquid metals, Phys. Rev. B 47, 558 (1993).

[48] G. Kresse and J. Hafner, Ab initio molecular-dynamics simulation of the liquid-metal-amorphous-semiconductor transition in germanium, Phys. Rev. B 49, 14251 (1994).
[49] G. Kresse and J. Furthmüller, Efficient iterative schemes for $a b$ initio total-energy calculations using a plane-wave basis set, Phys. Rev. B 54, 11169 (1996).

[50] G. Kresse and J. Furthmüller, Efficiency of ab-initio total energy calculations for metals and semiconductors using a plane-wave basis set, Comput. Mater. Sci. 6, 15 (1996).

[51] G. Bonny, N. Castin, C. Domain, P. Olsson, B. Verreyken, M. I. Pascuet, and D. Terentyev, Density functional theory-based cluster expansion to simulate thermal annealing in $\mathrm{FeCrW}$ alloys, Philos. Mag. 97, 299 (2017).

[52] J. M. Sanchez, Cluster expansion and the configurational theory of alloys, Phys. Rev. B 81, 224202 (2010).

[53] H. Okamoto, H-Zr (hydrogen-zirconium), J. Phase Equilib. Diffus. 27, 548 (2006).

[54] X. Zhu, D.-Y. Lin, J. Fang, X.-Y. Gao, Y.-F. Zhao, and H.-F Song, Structure and thermodynamic properties of zirconium hydrides by structure search method and first principles calculations, Comput. Mater. Sci. 150, 77 (2018). 